\title{
Targeting the CRP-HIF1a axis in RA improves response to leflunomide
}

In a substantial proportion of patients with rheumatoid arthritis (RA), treatment with the DMARD leflunomide fails to halt the progression of bone erosion, despite inhibiting inflammation. A new study reveals that $\mathrm{C}$-reactive protein (CRP)-hypoxia inducible factor $1 \alpha(\mathrm{HIF} 1 \alpha)$ signalling attenuates the response to leflunomide, and suggests that co-administration of a HIF1a inhibitor could improve the therapeutic response to leflunomide in selected patients.

"We performed binary logistic regression analysis to determine the relationships between the limited leflunomide response and commonly used clinical factors," says corresponding author Ge Zhang. "Surprisingly, we revealed that serum CRP concentrations showed predictive value for classifying the patients with RA with limited response to leflunomide."

In patients with RA and high serum concentrations of CRP (CRP-high), leflunomide attenuated

combined

treatment with

leflunomide and acriflavine, an FDA-

approved HIF 1 a inhibitor, prevented bone loss

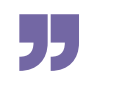

bone erosion to a lesser extent than in CRP-low patients; notably, leflunomide had similar immunomodulatory effects in both groups. Rats with collagen-induced arthritis (CIA) had similarly differential responsiveness to leflunomide treatment on the basis of CRP concentrations.

Further experiments revealed that in CRP-low rats, leflunomide

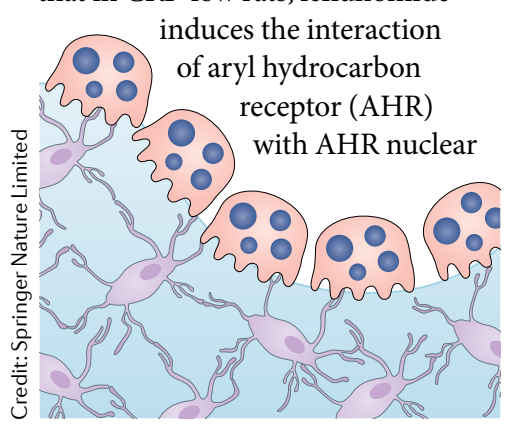

transloclator (ARNT) to inhibit hepatic CRP expression and thus attenuate bone erosion. In CRP-high rats, however, CRP upregulates expression of HIF1a, which competes with AHR for ARNT binding and interferes with leflunomide-AHRCRP signalling, ultimately limiting the response to leflunomide.

Knockdown of HIFla in vitro and hepatocyte-specific deletion of HIF1a in mice with CIA improved leflunomide-AHR-CRP signalling and, in the mice, inhibited bone erosion. Moreover, combined treatment with leflunomide and acriflavine, an FDA-approved HIF1a inhibitor, prevented bone loss in CRP-high rats with CIA.

Together, the results suggest that CRP could have clinical value for prediction of response to leflunomide treatment, and that the combination of leflunomide and acriflavine could be used as precision medicine for CRP-high patients with RA.

Sarah Onuora

ORIGINAL ARTICLE Liang, C. et al. HIF1 $\alpha$ inhibition facilitates leflunomide-AHR-CRP signaling to attenuate bone erosion in CRP-aberrant rheumatoid arthritis. Nat. Commun. 10, 4579 (2019)

\section{Liposomal targeting of DCs to induce tolerance}

New findings highlight targeting of dendritic cells (DCs) with antigen-containing liposomes as a promising strategy for inducing autoantigen-specific immunological tolerance in autoimmune diseases. "Our data show that immune regulation could be restored in inflammatory autoimmune diseases where autoantigen is widely expressed - in models of rheumatoid arthritis (RA) and renal vasculitis," reports corresponding author Ranjeny Thomas.

"Liposomes have an established clinical track record for targeted drug delivery and provide a platform for co-delivery to DCs of the lipophilic $\mathrm{NF}-\mathrm{\kappa B}$ inhibitor calcitriol with hydrophilic antigenic peptide," says Thomas. "Importantly, co-delivery of antigen in the liposomes ensures that immune regulation is antigen-specific and not generalized," explains Thomas.
Indeed, calcitriol-containing liposomes suppressed the cytokinesecreting and antigen-presenting capacity of DCs in vitro. In mice immunized with an antigenic peptide, administration of liposomes containing both calcitriol and the peptide suppressed the expansion of antigen-specific effector T cells and promoted the expansion of antigen-specific regulatory $T$ cells.

An important question was whether an inflammatory setting could inhibit the induction of tolerance by activating DCs. "We show that active inflammation actually supported tolerance induction with liposomes encapsulating peptide and calcitriol," reports Thomas. "Inflammation promoted PDL1 expression by DCs, PDL1+ DCs were more likely to take up the liposomes and PDL1 was required for the induction of regulatory T cells."
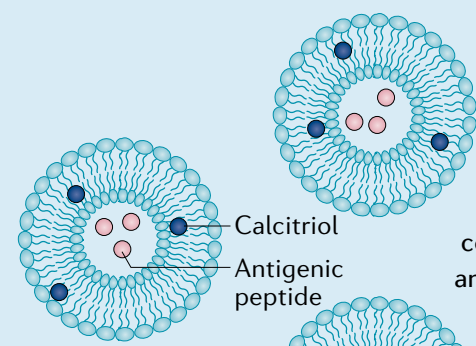

In mouse models of RA and Goodpasture's vasculitis (also known as anti-glomerular basement membrane disease), liposomes containing calcitriol and disease-associated peptides inhibited the development of autoimmunity and also suppressed existing disease.

Analysis using peptide-MHC class II tetramer staining to visualize the autoreactive $\mathrm{CD}^{+} \mathrm{T}$ cell compartment suggested that liposomal treatment decreased disease severity in an antigen-specific manner.

"The research opens up new potential for drug development and trials in patients with autoimmune diseases and in individuals at high risk of disease development," concludes Thomas.

Jessica McHugh

ORIGINAL ARTICLE Galea, R. et al. PD-L1- and calcitriol-dependent liposomal antigen-specific regulation of systemic inflammatory autoimmune disease. JCl Insight 4, e126025 (2019) 